\title{
Risk factors for severe cases of COVID-19: a retrospective cohort study
}

\author{
Feng $\mathrm{He}^{1,{ }^{*}}$, Qingqing Luo ${ }^{1, *}$, Ming Lei ${ }^{2,}{ }^{*}$, Lixin Fan², Xinning Shao², Guanglie Huang ${ }^{1}$, Jun Zeng ${ }^{1}$, \\ Ziwen Zhao', Shuguang Qin ${ }^{1}$, Zhi Yang ${ }^{1}$, Na Yu${ }^{1}$, Liuping Yang ${ }^{2, \&}$, Jie Cao ${ }^{1}$ \\ ${ }^{1}$ Guangzhou First People's Hospital, The Second Affiliated Hospital of South China University of Technology, \\ Guangzhou, China \\ ${ }^{2}$ Guangzhou Eighth People's Hospital, Guangzhou Medical University, Guangzhou, China \\ *Equal contribution
}

Correspondence to: Na Yu, Liuping Yang, Jie Cao; email: 546046726@qq.com, gz8hylp@126.com, eycaojie@scut.edu.cn Keywords: COVID-19, severe cases, risk factors, clinical characteristics

Received: April 18, $2020 \quad$ Accepted: July 9, 2020

Published: August 15, 2020

Copyright: He et al. This is an open-access article distributed under the terms of the Creative Commons Attribution License (CC BY 3.0), which permits unrestricted use, distribution, and reproduction in any medium, provided the original author and source are credited.

\section{ABSTRACT}

Background: SARS-CoV-2 has raged around the world since March, 2020. We aim to describe the clinical characteristics and risk factors of severe patients with COVID-19 in Guangzhou.

Results: The severity and mortality of COVID-19 was $10.4 \%$ and $0.3 \%$ respectively. And each 1-year increase in age (OR, 1.057; 95\% Cl, 1.018-1.098; $\mathrm{P}=0.004)$, Wuhan exposure history greater than 2 weeks (OR, 2.765; 95\% $\mathrm{Cl}$, 1.040-7.355; $\mathrm{P}=0.042$ ), diarrhea (OR, 24.349; 95\% Cl, 3.580-165.609; $\mathrm{P}=0.001$ ), chronic kidney disease (OR, 6.966; 95\% Cl, 1.310-37.058; $\mathrm{P}=0.023)$, myoglobin higher than $106 \mu \mathrm{g} / \mathrm{L}(\mathrm{OR}, 8.910 ; 95 \% \mathrm{Cl}, 1.225-64.816$; $\mathrm{P}=0.031$ ), white blood cell higher than $10 \times 10^{9} / \mathrm{L}$ (OR, 5.776; $\left.95 \% \mathrm{Cl}, 1.052-31.722 ; \mathrm{P}=0.044\right)$, and C-reactive protein higher than $10 \mathrm{mg} / \mathrm{L}(\mathrm{OR}, 5.362 ; 95 \% \mathrm{Cl}, 1.631-17.626 ; \mathrm{P}=0.006)$ were risk factors for severe cases.

Conclusion: Older age, Wuhan exposure history, diarrhea, chronic kidney disease, elevated myoglobin, elevated white blood cell and C-reactive protein were independent risk factors for severe patients with COVID-19 in Guangzhou.

Methods: We included 288 adult patients with COVID-19 and compared the data between severe and non-severe group. We used univariate and multivariate logistic regression methods to explore risk factors of severe cases.

\section{INTRODUCTION}

In December 2019, a large-scale infectious pneumonia of unknown origin broke out in Wuhan, China. Chinese scientists isolated a new coronavirus, SARS-CoV-2, causing the pneumonia on Jan 7, $2020[1,2]$. And WHO named it Coronavirus Disease 2019 (COVID-19) in February 2020 [3]. Since March, justifying the previous data model [4], COVID-19 has raged across world. Up to Jun 3, 2020, there have been more than 6.4 million diagnosed cases in more than 200 countries, with a mortality rate of about $6 \%$ [5].
The clinical manifestations of COVID-19 range from mild to critical [6]. A lot of observational studies have described the clinical characteristics of patients with COVID-19 in Wuhan [7-10], but studies outside Wuhan have rarely been reported. Because of the virus variation, the clinical characteristics of the patients in Wuhan and outside Wuhan maybe different. In this study, we aimed to investigate patients with COVID-19 in Guangzhou to find their clinical characteristics and the risk factors for severe cases. Monitoring these factors can help clinicians identify severe patients early and take subsequent interventions to reduce their illness. 


\section{RESULTS}

\section{Baseline characteristics}

Among the 288 patients, 30 cases were in severe group and only 1 case died by the end of the study. Thus, the severity and mortality were $10.4 \%$ and $0.3 \%$ respectively. The median age of all patients was 48.5 years (IQR 34.3-62), of which women accounted for $54.5 \% \quad$ (Table 1). $134 \quad(46.5 \%)$ patients had comorbidities, of which cardiovascular disease (CVD) $(85,29.5 \%)$ was the most common one, followed by hypertension $(84,29.2 \%)$, diabetes $(24,8.3 \%)$ (Table 1). 132 patients $(45.8 \%)$ had a history of exposure to Wuhan 2 weeks before onset (Table 1). The most common symptoms on admission were fever (201, $69.8 \%)$ and cough $(163,56.6 \%)$, followed by sputum $(58,20.1 \%)$, fatigue $(43,14.9 \%)$, and myalgia (35, 12.2\%) (Table 1).

\section{Laboratory and radiological findings}

$216(75 \%)$ patients had white blood cells (WBC) in normal range and lymphopenia occurred in 91 (31.6\%) patients (Table 2). Compared with non-severe patients, severe patients had significantly reduced serum hemoglobin, platelet and myoglobin, as well as significantly increased WBC, alanine aminotransferase (ALT), aspartate aminotransferase (AST), creatinine, creatine kinase, $\mathrm{C}$-reactive protein (CRP), procalcitonin (PCT), brain natriuretic peptide (BNP), and troponin I (Table 2). 31 (10.8\%) patients had unilateral pneumonia, and all of them were non-severe patients; $241(83.7 \%)$ patients had bilateral pneumonia, of which 29 (96.7\%) were severe patients (Table 2). Their chest CTs showed varying degrees of patchy ground-glass opacity, with lung lesion area of severe patients usually larger than that of non-severe patients (Figure 1).

\section{Treatments and outcomes}

$244(84.7 \%)$ patients received antibiotics, and 233 (80.9\%) patients received antiviral drugs (oseltamivir / ribavirin; Table 3). There was a significant difference in the use of glucocorticoids and vasoactive drugs between non-severe and severe patients (Table 3). Five patients were treated with continuous renal replacement therapy (CRRT) and four patients were treated with extracorporeal membrane oxygenation (ECMO), and they were all severe patients (Table 3). $98.9 \%$ of the non-severe patients did not take oxygen or took normalflux oxygen, while $43.3 \%$ of the severe patients took high-flux oxygen (Table 3 ). Eight patients were tracheal intubated and they were all severe patients (Table 3). Severe patients had a significant increase in use of noninvasive mechanical ventilation than non-severe patients (Table 3). Compared with non-severe patients, severe patients were more likely to be transferred to the intensive care unit (ICU), and suffer from ARDS, acute kidney injury and acute cardiac injury (Table 3 ).

\section{Univariate and multivariate analysis of risk factors of severe cases}

In univariate logistic regression analysis, we found that older patients with hypertension, chronic kidney disease (CKD), and a history of exposure in Wuhan were more likely to develop severe disease (Table 4). In addition, fever, shortness of breath, diarrhea, WBC, CRP, lymphocytes, COPD, CVD, hemoglobin, ALT, AST, myoglobin, creatinine, creatine kinase, PCT, BNP and TNI were also related with severe cases (Table 4).

The multivariable logistic regression model was constructed using all variables of significant statistical differences in univariate logistic regression analysis. We found that each 1-year increase in age (OR, 1.057; 95\% CI, 1.018-1.098; $\mathrm{P}=0.004)$, Wuhan exposure history greater than 2 weeks (OR, 2.765; 95\% CI, 1.040-7.355; $\mathrm{P}=0.042)$, CKD (OR, 6.966; 95\% CI, 1.310-37.058; $\mathrm{P}=0.023$ ), diarrhea (OR, 24.349; $95 \%$ CI, 3.580-165.609; $\mathrm{P}=0.001)$, Myoglobin higher than $106 \mu \mathrm{g} / \mathrm{L}$ (OR, 8.910; 95\% CI, 1.225-64.816; $\mathrm{P}=0.031$ ), WBC higher than $10 \times 10^{9} / \mathrm{L}(\mathrm{OR}, 5.776$; $95 \% \mathrm{CI}, 1.052-$ 31.722; $\mathrm{P}=0.044$ ), and CRP higher than $10 \mathrm{mg} / \mathrm{L}$ (OR, 5.362; 95\% CI, 1.631-17.626; $\mathrm{P}=0.006)$ were independent risk factors for severe cases (Table 4).

\section{DISCUSSION}

Of the 288 patients in our database, only one case $(0.3 \%)$ died, while the early mortality rate in Wuhan was as high as $28.3 \%$ [3]. And the severity of COVID19 in Guangzhou is $10.4 \%$, which was far less than that in early Wuhan of $31.7 \%$ [11].

It was interesting to note Guangzhou patients with Wuhan exposure history had a higher risk of becoming severe cases (Table 4). Earlier reports reported that some patients had SARS-CoV-2 gene fragments missing, suggesting that their virulence gradually weakened [12]. And an article reported that COVID-19 patients in Zhejiang Province had relatively mild symptoms compared with Wuhan [13]. Later, it was reported that SARS-CoV-2 has genomic diversity. It mutated through replication and may evolve under the pressure of immune surveillance in human body, with its virulence, infectivity and transmission being affected [14]. Therefore, the virulence of SARS-CoV-2 may increase or decrease during transmission, and certain populations in different regions may also have a screening effect on it, resulting in different disease 
Table 1. Baseline characteristics of non-severe or severe patients of COVID-19 in Guangzhou.

\begin{tabular}{|c|c|c|c|c|}
\hline \multirow{2}{*}{$\begin{array}{l}\text { Demographics and clinical } \\
\text { characteristics }\end{array}$} & \multicolumn{3}{|c|}{ No. $(\%)$} & \multirow{2}{*}{$P$ value } \\
\hline & Total (288) & Non-severe (258) & Severe (30) & \\
\hline Age, median (IQR), years & $48.5(34.3-62)$ & $47(33-61)$ & $61.5(51-71.3)$ & $<0.0001$ \\
\hline Age groups (years): &.. &.. & .. & $<0.0001$ \\
\hline$\leq 30$ & $44(15.3)$ & $44(17.1)$ & $0(0)$ &.. \\
\hline $31-45$ & $87(30.2)$ & $83(32.2)$ & $4(13.3)$ & .. \\
\hline $46-65$ & $116(40.3)$ & $101(39.1)$ & $15(50)$ & .. \\
\hline$\geq 66$ & $41(14.2)$ & $30(11.6)$ & $11(36.7)$ &.. \\
\hline Sex: &.. & .. & .. & 0.194 \\
\hline Male & $131(45.5)$ & $114(44.2)$ & $17(56.7)$ &.. \\
\hline Female & $157(54.5)$ & $114(55.8)$ & $13(43.3)$ &.. \\
\hline Comorbidity: &.. &.. &.. &.. \\
\hline Hypertension & $84(29.2)$ & $69(26.7)$ & $15(50)$ & 0.008 \\
\hline SBP (mm Hg), median (IQR) & $125(117-136)$ & $125(117-136)$ & $124.5(117-138.3)$ & 0.186 \\
\hline DBP (mm Hg), median (IQR) & $80(74-87)$ & $80(75-87)$ & $80.5(67.3-85)$ & 0.028 \\
\hline MAP (mm Hg), median (IQR) & $94.7(87.8-103)$ & $94.7(88-103)$ & $94.8(85.1-102.6)$ & 0.415 \\
\hline Diabetes & $24(8.3)$ & $20(7.8)$ & $4(13.3)$ & 0.295 \\
\hline COPD & $5(1.7)$ & $3(1.2)$ & $2(6.9)$ & 0.025 \\
\hline CVD & $85(29.5)$ & $70(27.1)$ & $15(50)$ & 0.009 \\
\hline Carcinoma & $6(2.1)$ & $6(2.3)$ & $0(0)$ & 0.399 \\
\hline CKD & $8(2.8)$ & $4(1.6)$ & $4(13.3)$ & $<0.0001$ \\
\hline CLD & $10(3.5)$ & $8(3.1)$ & $2(6.7)$ & 0.313 \\
\hline Exposure history in Wuhan $>2$ weeks: &.. &.. &.. & 0.016 \\
\hline Yes & $132(45.8)$ & $112(43.4)$ & $20(66.7)$ &.. \\
\hline No & $156(54.2)$ & $146(56.6)$ & $10(33.3)$ & .. \\
\hline Respiratory rate $>24$ breaths per min & 19(6.6) & $12(4.7)$ & $7(23.3)$ & $<0.0001$ \\
\hline Oxygenation index, median (IQR) & $98(97-98.8)$ & $98(97-98.8)$ & $98(97-99)$ & 0.986 \\
\hline Fever (tempetature $\geq 37 \cdot 3^{\circ} \mathrm{C}$ ) & $201(69.8)$ & $174(67.4)$ & $27(90)$ & 0.011 \\
\hline Cough & $163(56.6)$ & $142(55)$ & $21(70)$ & 0.118 \\
\hline Sputum & $58(20.1)$ & $54(20.9)$ & $4(13.3)$ & 0.326 \\
\hline Myalgia & $35(12.2)$ & $30(11.6)$ & $5(16.7)$ & 0.424 \\
\hline Fatigue & $43(14.9)$ & $37(14.3)$ & $6(20)$ & 0.410 \\
\hline Nausea or Anorexia & $28(9.7)$ & $22(8.5)$ & $6(20)$ & 0.045 \\
\hline Vomiting & $6(2.1)$ & $5(1.9)$ & $1(3.3)$ & 0.613 \\
\hline Diarrhea & $11(3.8)$ & $6(2.3)$ & $5(16.7)$ & $<0.0001$ \\
\hline Headache & $26(9)$ & $22(8.5)$ & $4(13.3)$ & 0.385 \\
\hline
\end{tabular}

SBP, Systolic blood pressure; DBP, Diastolic blood pressure; MAP, Mean arterial pressure; COPD, Chronic obstructive pulmonary disease; CVD, Cardiovascular disease; CKD, Chronic kidney disease; CLD, Chronic liver disease.

* $\boldsymbol{P}$ values indicate differences between Severe and Non-severe patients. $P<0.05$ was considered statistically significant.

Table 2. Laboratory and radiological findings of non-severe or severe patients of COVID-19 in Guangzhou.

\begin{tabular}{lccccc}
\hline & \multicolumn{3}{c}{ Median (IQR) } & P value & $\begin{array}{c}\text { Normal } \\
\text { range }\end{array}$ \\
\cline { 2 - 5 } & Total (288) & Non-severe (258) & Severe (30) & & \\
\hline Laboratory findings & & & & & \\
WBC $\left(\times 10^{9} / \mathrm{L}\right)$ & $5.20(4.14-6.44)$ & $5.14(4.10-6.38)$ & $5.33(4.42-7.18)$ & 0.934 & $4-10$ \\
WBC $\left(\times 10^{9} / \mathrm{L}\right)($ No $(\%)):$ &.. &.. &.. & $<0.0001$ &.. \\
$\quad<4$ & $62(21.5)$ & $57(22.1)$ & $5(16.7)$ &.. &.. \\
& & & & \\
\hline
\end{tabular}




\begin{tabular}{|c|c|c|c|c|c|}
\hline $4-10$ & $216(75)$ & $197(76.4)$ & $19(63.3)$ & .. & .. \\
\hline$>10$ & $10(3.5)$ & $4(1.6)$ & $6(20)$ & .. & .. \\
\hline Lymphocyte count $\left(\times 10^{9} / \mathrm{L}\right)$ & $1.42(1.04-1.96)$ & $1.46(1.09-1.97)$ & $1.03(0.84-1.38)$ & 0.511 & $1.1-3.2$ \\
\hline $\begin{array}{l}\text { Lymphocyte count }\left(\times 10^{9} / \mathrm{L}\right) \text { (No } \\
(\%)) \text { : }\end{array}$ & 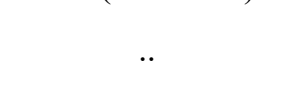 & 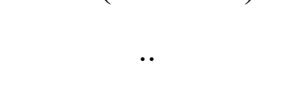 & 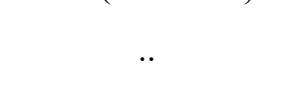 &.$\cdot$ & .. \\
\hline$<1.1$ & 91(31.6) & $73(28.3)$ & $18(60)$ & $<0.0001$ & \\
\hline Hemoglobin $(\mathrm{g} / \mathrm{L})$ & $135.5(123-147)$ & $136(125-147.3)$ & $123(114-143.3)$ & 0.001 & $130-175$ \\
\hline Platelet count $\left(\times 10^{9} / \mathrm{L}\right)$ & $194.5(158-247)$ & $199(160.1-249.3)$ & $167(140.3-188.5)$ & 0.043 & $125-350$ \\
\hline D-dimer $(\mathrm{mg} / \mathrm{L})$ & $1110(700-1700)$ & $1090(680-1600)$ & $1855(865-3442.5)$ & 0.052 & $<1000$ \\
\hline D-dimer (mg/L) (No (\%)): & .. & .. & .. & 0.106 &.. \\
\hline$\leq 1000$ & $125(43.9)$ & $116(45.5)$ & $9(30)$ &.. & .. \\
\hline$>1000$ & $160(56.1)$ & $139(54.5)$ & $21(70)$ &.. &.. \\
\hline $\operatorname{ALT}(\mathrm{U} / \mathrm{L})$ & $22.5(14.3-34.5)$ & $22.1(14.2-33.9)$ & $25(16.1-49.1)$ & 0.912 & $9-50$ \\
\hline $\operatorname{ALT}(\mathrm{U} / \mathrm{L})(\mathrm{No}(\%))$ : & .. & .. & .. & 0.039 &.. \\
\hline$\leq 50$ & $254(88.2)$ & $231(89.5)$ & $23(76.7)$ &.. & .. \\
\hline$>50$ & $34(11.8)$ & $27(10.5)$ & $7(23.3)$ & .. &.. \\
\hline $\operatorname{AST}(\mathrm{U} / \mathrm{L})$ & $18.4(14.9-25.6)$ & $18.1(14.5-24.5)$ & $21.9(16.8-41.1)$ & 0.161 & $15-40$ \\
\hline $\operatorname{AST}(\mathrm{U} / \mathrm{L})(\mathrm{No}(\%))$ : & .. & .. & .. & 0.004 &.. \\
\hline$\leq 40$ & $256(88.9)$ & $234(90.7)$ & $22(73.3)$ &.. & .. \\
\hline$>40$ & $32(11.1)$ & $24(9.3)$ & $8(26.7)$ & .. & .. \\
\hline Myoglobin $(\mu \mathrm{g} / \mathrm{L})$ & $15(8.85-22.4)$ & $14.4(8.6-21.2)$ & $27.3(13.1-86.6)$ & 0.212 & $17.4-105.7$ \\
\hline Myoglobin $(\mu \mathrm{g} / \mathrm{L})(\mathrm{No}(\%))$ : & .. & .. & .. & $<0.0001$ & .. \\
\hline$\leq 106$ & $269(97.1)$ & 247(99.2) & $22(78.6)$ & .. & .. \\
\hline$>106$ & $8(2.9)$ & $2(0.8)$ & $6(21.4)$ &.. &.. \\
\hline Creatinine $(\mu \mathrm{mol} / \mathrm{L})$ & $61.8(50.25-76.56)$ & $62.0(50.4-76.4)$ & $59.6(45.9-78.1)$ & 0.428 & $54-106$ \\
\hline Creatinine $(\mu \mathrm{mol} / \mathrm{L})(\mathrm{No}(\%))$ : & .. & .. & .. & 0.022 &.. \\
\hline$\leq 106$ & $279(96.9)$ & $252(97.7)$ & $27(90)$ &.. & .. \\
\hline$>106$ & $9(3.1)$ & $6(2.3)$ & $3(10)$ & .. & .. \\
\hline Creatinine kinase (U/L) & $52(36-80)$ & $52(37-80)$ & $44.5(27.5-128)$ & 0.238 & $50-310$ \\
\hline Creatinine kinase (U/L) (No (\%)): & .. & .. & .. & 0.009 &.. \\
\hline$\leq 310$ & $283(98.6)$ & $255(99.2)$ & $28(93.3)$ &.. & .. \\
\hline$>310$ & $4(1.4)$ & $2(0.8)$ & $2(6.7)$ & .. & .. \\
\hline $\mathrm{CRP}(\mathrm{mg} / \mathrm{L})$ & $9(8-22.72)$ & $9(8-18.9)$ & $24(11.7-51.2)$ & 0.005 & $<10$ \\
\hline CRP $(\mathrm{mg} / \mathrm{L})(\mathrm{No}(\%))$ : & .. & .. & .. & $<0.0001$ &.. \\
\hline$\leq 10$ & $175(60.8)$ & $169(65.5)$ & $6(20)$ & .. & .. \\
\hline$>10$ & $113(39.2)$ & $89(34.5)$ & $24(80)$ & .. & .. \\
\hline $\mathrm{PCT}(\mathrm{ng} / \mathrm{mL})$ & $0.13(0.04-32.6)$ & $0.106(0.035-32.58)$ & $0.2(0.09-51)$ & 0.241 & $<0.05$ \\
\hline PCT (ng/mL) (No (\%)): & .. & .. & .. & $<0.0001$ &.. \\
\hline$<0.05$ & $99(35.5)$ & $96(38.4)$ & $3(10.3)$ & .. & .. \\
\hline $0.05-1.0$ & $73(26.2)$ & $56(22.4)$ & $17(58.6)$ & .. & .. \\
\hline $1 . .0-10$ & $6(2.2)$ & $6(2.4)$ & $0(0)$ & .. & .. \\
\hline$>10$ & $101(36.2)$ & $92(36.8)$ & $9(31)$ &.. &.. \\
\hline BNP (ng/L) & $35(13-117.5)$ & $18.5(9.75-40.25)$ & 213(45-399) & 0.014 & $<100$ \\
\hline TNI $(\mu \mathrm{g} / \mathrm{L})$ & $0.004(0.001-0.009)$ & $0.003(0.001-0.007)$ & $0.027(0.010-0.099)$ & 0.033 & $<0.03$ \\
\hline TNI $(\mu \mathrm{g} / \mathrm{L})(\mathrm{No}(\%))$ : & .. & .. & .. & $<0.0001$ &.. \\
\hline$\leq 0.03$ & $168(88.4)$ & $159(92.4)$ & $9(50)$ & .. & .. \\
\hline$>0.03$ & $22(11.6)$ & $13(7.6)$ & $9(50)$ & .. & .. \\
\hline \multicolumn{6}{|l|}{ Chest radiography findings } \\
\hline Unilateral pneumonia & $31(10.8)$ & $31(12.1)$ & $0(0)$ & 0.044 & .. \\
\hline Bilateral pneumonia & $241(83.7)$ & $212(82.2)$ & $29(96.7)$ & 0.042 & .. \\
\hline
\end{tabular}

WBC, White blood cell; ALT, Alanine transaminase; AST, Aspartate aminotransferase; CRP, C-reactive protein; PCT, Procalcitonin; BNP, Brain natriuretic peptide; TNI, Troponin I.

${ }^{*} \boldsymbol{P}$ values indicate differences between Severe and Non-severe patients. $P<0.05$ was considered statistically significant. 
degrees and influencing factors of COVID-19 in different regions.

According to previous reports, older age was an important independent predictor of SARS and MERS mortality $[15,16]$. Previous studies have confirmed increased severity and mortality of COVID-19 in old patients $[3,7,17]$. A recent study comparing the clinical characteristics and results of COVID-19 patients of different ages showed that the symptoms of elderly patients were more atypical, with more comorbidities, secondary infection, organ injuries, immunodeficiency and a higher risk of critical illness [18]. Many comorbidities in the elderly such as hypertension, diabetes and CKD were treated with ACE inhibitors and angiotensin II receptor blockers, which would upregulate the ACE2 receptor, thereby increasing the risk of SARS-CoV-2 infection and the risk of disease
[19]. In our study cohort, age was also one of the risk factors for severe patients (Table 4). Therefore, it's very important for old patients to have early diagnosis and treat systemic comorbidities carefully.

SARS-CoV-2 was reported to be detected in stool samples from patients [20], and a study of a family cluster have reported two COVID-19 patients who had only diarrhea symptom [9]. Besides diarrhea, some patients also had other gastrointestinal symptoms such as vomiting and abdominal pain [21]. Our analysis showed that diarrhea was a risk factor for severe cases (Table 4), which suggested that beside of damaging the respiratory system, the virus may also have a certain function on the digestive system. This finding may be related to the expression of SARS-CoV-2 receptor ACE2 in both the epithelial cells of lungs and digestive tract [21, 22]. Given the small number of diarrhea cases

Case 1
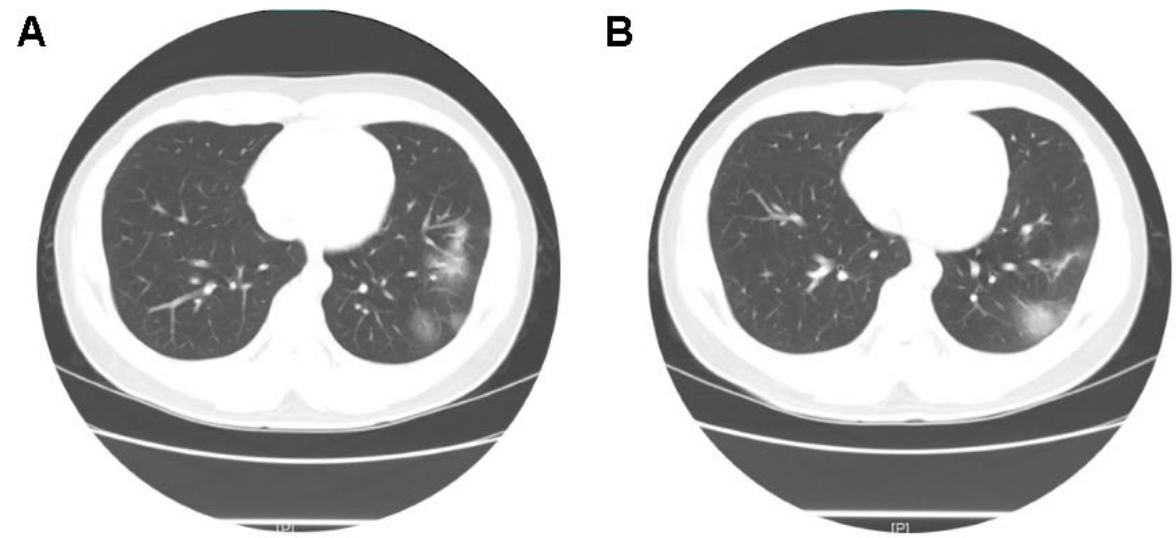

Case 2
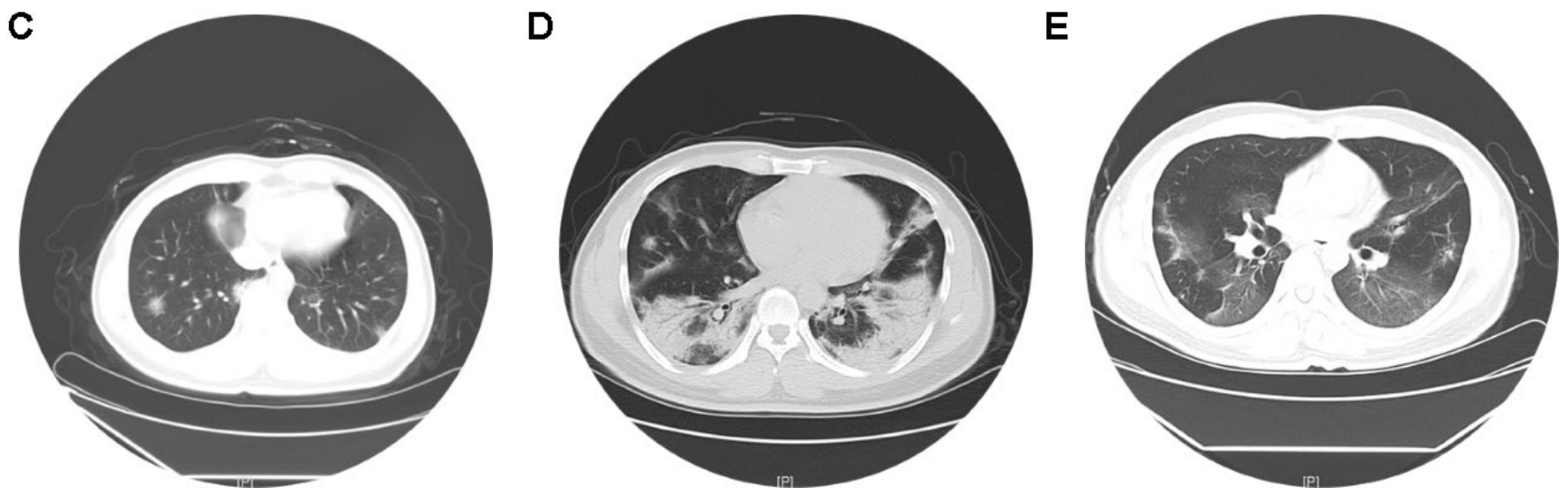

Figure 1. Chest CTs of two representative cases. Case 1 (non-severe): Chest CT on Feb 24 (A) showed multiple patchy ground-glass opacity in both lungs, with unclear borders and uneven density. Chest CT on Feb 28 (B) showed better status, and some lesions were slightly absorbed than before. Case 2 (severe): Chest CT on Jan 29 (C) showed the texture of both lungs was slightly increased, and both lungs were scattered in patchy shadows, whose edges were blurred. Chest CT on Feb 11 (D) showed the scope of the bilateral lung lesions was enlarged, the density was increased, and the local consolidation and bronchial signs were seen. Chest CT on Mar 4 (E) showed improved status, and both lung lesions were significantly less than before. 
Table 3. Treatments and outcomes of non-severe or severe patients of COVID-19 in Guangzhou.

\begin{tabular}{lcccc}
\hline & \multicolumn{3}{c}{ No. (\%) } & \multirow{2}{*}{ P value } \\
\cline { 2 - 3 } & Total (288) & Non-severe (258) & Severe (30) & \\
\hline Treatments & & & & 0.020 \\
Antiviral & $233(80.9)$ & $204(79.1)$ & $29(96.7)$ & 0.014 \\
Antibiotics & $244(84.7)$ & $214(82.9)$ & $30(100)$ & $<0.0001$ \\
Vasoactive drugs & $5(1.7)$ & $1(0.4)$ & $4(13.3)$ & $<0.0001$ \\
Glucocorticoid & $21(7.3)$ & $12(4.7)$ & $9(30)$ & $<0.0001$ \\
CRRT & $5(1.7)$ & $0(0)$ & $5(16.7)$ & $<0.0001$ \\
ECMO & $4(1.4)$ & $0(0)$ & $4(13.3)$ &.. \\
Oxygen uptake: &. &.. &.. & 0.030 \\
None & $88(30.6)$ & $84(32.6)$ & $4(13.3)$ & 0.013 \\
Normal-flux & $184(63.9)$ & $171(66.3)$ & $13(43.3)$ & $<0.0001$ \\
High-flux & $16(5.6)$ & $3(1.2)$ & $8(26.7)$ & $<0.0001$ \\
Tracheal intubation & $8(2.8)$ & $0(0)$ & $0(0)$ &.. \\
Tracheotomy & $0(0)$ & $0(0)$ & $19(63.3)$ & $<0.0001$ \\
Non-invasive mechanical ventilation & $32(11.1)$ & $13(5)$ & & \\
Outcomes & & & $15(50)$ & $<0.0001$ \\
ICU Admission & $27(9.4)$ & $12(4.7)$ & $3(10)$ & $<0.0001$ \\
ARDS & $3(1)$ & $0(0)$ & $5(16.7)$ & $<0.0001$ \\
Acute kidney injury & $5(1.7)$ & $0(0)$ & $9(50)$ & $<0.0001$ \\
Acute cardiac injury & $22(11.6)$ & $13(7.6)$ & & \\
\hline
\end{tabular}

CRRT, continuous renal-replacement therapy; ECMO, Extracorporeal membrane oxygenation; ICU, intensive care unit; ARDS, Acute respiratory distress syndrome.

${ }^{*} \boldsymbol{P}$ values indicate differences between Severe and Non-severe patients. $\boldsymbol{P}<0.05$ was considered statistically significant.

Table 4. Univariate and multivariate analysis of risk factors of severe cases in Guangzhou.

\begin{tabular}{|c|c|c|c|c|}
\hline & Univariable OR $(95 \% \mathrm{CI})$ & P value & Multivariable OR (95\%) CI) & P value \\
\hline \multicolumn{5}{|l|}{ Demographics and clinical characteristics } \\
\hline Age, years & $1.063(1.033-1.095)$ & $<0.0001$ & $1.057(1.018-1.098)$ & 0.004 \\
\hline Female sex (vs male) & $0.605(0.282-1.298)$ & 0.197 & .. &.. \\
\hline \multicolumn{5}{|l|}{ Comorbidity present (vs not present) } \\
\hline Hypertension & $2.739(1.272-5.898)$ & 0.010 & .. & .. \\
\hline COPD & $6.296(1.007-39.354)$ & 0.049 &.. &.. \\
\hline CVD & $2.686(1.248-5.780)$ & 0.012 & $0.986(0.052-18.588)$ & 0.992 \\
\hline CKD & $9.769(2.307-41.376)$ & 0.002 & $6.966(1.310-37.058)$ & 0.023 \\
\hline Respiratory rate $>24$ breaths per min & $6.239(2.238-17.397)$ & $<0.0001$ & .. &.. \\
\hline Exposure history in Wuhan $>2$ weeks & $2.607(1.174-5.791)$ & 0.019 & $2.765(1.040-7.355)$ & 0.042 \\
\hline Fever (tempetature $\left.\geq 37 \cdot 3^{\circ} \mathrm{C}\right)$ & $4.345(1.282-14.730)$ & 0.018 & .. &.. \\
\hline Nausea or Anorexia & $2.682(0.991-7.258)$ & 0.052 & .. &.. \\
\hline Diarrhea & $8.400(2.392-29.494)$ & 0.001 & $24.349(3.580-165.609)$ & 0.001 \\
\hline \multicolumn{5}{|l|}{ Laboratory and radiography findings } \\
\hline White blood cell count $\left(10^{9} / \mathrm{L}\right)$ (No (\%)): & .. &.. & .. &.. \\
\hline$\leq 4$ & $0.910(0.325-2.543)$ & 0.857 & $0.968(0.289-3.245)$ & 0.958 \\
\hline $4-10$ & $1(\mathrm{ref})$ &.. & .. &.. \\
\hline$\geq 10$ & $15.553(4.032-59.988)$ & $<0.0001$ & $5.776(1.052-31.722)$ & 0.044 \\
\hline Lymphocyte count $\left(\times 10^{9} / \mathrm{L}\right)$ & .. &.. & .. &.. \\
\hline$<1.1$ & $0.263(0.121-0.573)$ & 0.001 & $0.697(0.246-1.975)$ & 0.497 \\
\hline
\end{tabular}




\begin{tabular}{|c|c|c|c|c|}
\hline Hemoglobin $(\mathrm{g} / \mathrm{L})$ & $0.966(0.946-0.986)$ & 0.001 & .. & .. \\
\hline Platelet count $\left(\times 10^{9} / \mathrm{L}\right)$ & $0.993(0.987-1.000)$ & 0.042 & .. & .. \\
\hline D-dimer $(\mathrm{mg} / \mathrm{L})$ &.. &.. & .. & .. \\
\hline$\leq 1000$ & 1 (ref) & .. & .. & .. \\
\hline$>1000$ & $1.947(0.859-4.416)$ & 0.111 & .. & .. \\
\hline $\operatorname{ALT}(\mathrm{U} / \mathrm{L})$ &.. &.. & .. & .. \\
\hline$\leq 50$ & $1(\mathrm{ref})$ &.. & .. & .. \\
\hline$>50$ & $2.604(1.022-6.634)$ & 0.045 & .. & .. \\
\hline AST(U/L) & .. & .. & .. & .. \\
\hline$\leq 40$ & $1(\mathrm{ref})$ &.. & .. & .. \\
\hline$>40$ & $3.545(1.425-8.823)$ & 0.007 & .. & .. \\
\hline Myoglobin $(\mu \mathrm{g} / \mathrm{L})$ &.. &.. & .. & .. \\
\hline$\leq 106$ & 1 (ref) & .. & .. & .. \\
\hline$>106$ & $33.682(6.413-176.905)$ & $<0.0001$ & $8.910(1.225-64.816)$ & 0.031 \\
\hline Creatinine $(\mu \mathrm{mol} / \mathrm{L})$ &.. & .. &.. &.. \\
\hline$\leq 106$ & $1(\mathrm{ref})$ &.. & .. & .. \\
\hline$>106$ & $4.667(1.104-19.728)$ & 0.036 & .. & .. \\
\hline Creatinine kinase $(\mathrm{U} / \mathrm{L})$ &.. &.. & .. & .. \\
\hline$\leq 310$ & 1 (ref) & .. & .. & .. \\
\hline$>310$ & $9.107(1.234-67.188)$ & 0.030 & .. & .. \\
\hline CRP (mg/L) & .. &.. & .. & .. \\
\hline$\leq 10$ & $1(\mathrm{ref})$ &.. &.. &.. \\
\hline$>10$ & $7.596(2.995-19.264)$ & $<0.0001$ & $5.362(1.631-17.626)$ & 0.006 \\
\hline $\mathrm{PCT}(\mathrm{ng} / \mathrm{mL})$ &.. &.. & .. & .. \\
\hline$\leq 0.05$ & 1 (ref) &.. & .. & .. \\
\hline$>0.05$ & $5.333(1.572-18.098)$ & 0.007 & .. & .. \\
\hline BNP (ng/L) & $1.022(1.005-1.040)$ & 0.014 & .. & .. \\
\hline TNI $(\mu \mathrm{g} / \mathrm{L})$ &.. &.. & .. & .. \\
\hline$\leq 0.03$ & $1(\mathrm{ref})$ & .. & .. & .. \\
\hline$>0.03$ & $12.231(4.14-36.131)$ & $<0.0001$ & .. &.. \\
\hline Bilateral pneumonia & $6.292(0.836-47.378)$ & 0.074 & .. & .. \\
\hline
\end{tabular}

$\mathrm{OR}=$ odds ratio.

${ }^{*} \boldsymbol{P}$ values indicate differences between Severe and Non-severe patients. $\mathrm{P}<0.05$ was considered statistically significant.

$(11,3.8 \%)$ (Table 1), SARS-CoV-2-induced digestive system damage may also be related to other physical factors of these patients, which deserves further study.

Previous studies have reported that COVID-19 nonsurvivors had more neutrophil counts than survivors, which may be related to cytokine storms caused by virus invasion $[7,11]$. Our analysis found that elevated WBC and CRP were risk factors for severe cases (Table 4). Like neutrophils, WBC and CRP are also indicators of inflammatory status in the body. When they elevated, there may be a cytokine storm caused by virus invasion in the body, which may cause severe inflammation in lungs and other organs, and aggravate the disease. Therefore, paying close attention to changes in $\mathrm{WBC}$, CRP and making timely correction can effectively reduce the number of severe cases and deaths.
In our study, myoglobin, creatine kinase, BNP, and TNI were increased in severe patients compared to non-severe patients (Table 2), and myoglobin was a risk factor for severe patients, which indicated that COVID-19 may be related to acute cardiac injury. ACE2 is also expressed in heart [23], and SARS-CoV has been shown in animal models to directly mediate myocardial inflammation and damage by downregulating myocardial ACE2 and lead to poor cardiac prognosis [24]. A meta-analysis involving 4189 patients showed that more severe COVID-19 was associated with increased troponin, creatine kinase, myoglobin, and NT-proBNP [25]. Myoglobin was also included in the COVID-19 severity score table as one of the biomarkers [26]. The severity of COVID-19 may be related to acute cardiac injury, which prompts us to effectively monitor heart condition to prevent 
COVID-19 patients from myocarditis and avoid poor cardiac prognosis.

Many studies have reported that comorbidities were major risk factors for increasing COVID mortality and poor prognosis $[7,8]$, and CKD was one of them. Due to older age, previous comorbidities, impaired immune system, and regular visits to crowded outpatient dialysis centers, CKD patients have increase susceptibility to SARS-COV-2 [27]. On one hand, the above factors have greatly reduced the ability of CKD patients to overcome the virus and may lead to severe disease or even death. On the other hand, SARS-COV-2 can directly damage kidney by combine with ACE2 [28], and cause kidney inflammation and acute kidney injury $[13,29]$, which was consistent with the increase creatinine level of severe patients in our study. AKI could further aggravate CKD as well as worsening the patients' whole conditions, leading patients to develop severe illness.

The study has several limitations. First, the sample size of our study cohort was relatively small including only 288 patients from a single center. Due to the exploratory nature of the study, which was not driven by formal hypotheses, we did not estimate the sample size, but included as many cases as possible. Second, this study lacked laboratory data such as serum cytokines and chemokines, so that we cannot evaluate the inflammation levels and cytokine storms of these patients. Third, this was a retrospective study. The data in this study was only a preliminary assessment of clinical characteristics and risk factors of COVID-19 severe patients. Further researches are still needed.

In conclusion, our research showed that the severity and mortality of COVID-19 in Guangzhou were much lower than those in early Wuhan. The risk factors for severe cases of COVID-19 in Guangzhou included older age, Wuhan exposure history greater than 2 weeks, diarrhea, elevated Myoglobin, elevated $\mathrm{WBC}$ and CRP, and $\mathrm{CKD}$. Investigating and monitoring these factors can help clinicians identify patients with poor prognosis at an early stage, and take proactive interventions to benefit patients and reduce severity and mortality. It also provided significant experience and reference for countries around the world to fight against COVID-19.

\section{MATERIALS AND METHODS}

\section{Study design and participants}

This single-center, retrospective cohort study was conducted at Guangzhou Eighth People's Hospital (Guangzhou, China), which was the designated hospital to treat patients with COVID-19 in Guangzhou. From
Jan 15, 2020 to Mar 10, 2020, we recruited 288 adult patients with COVID-19 (the total number was 292, including 4 underage patients).

This study was approved by the Ethics Committee of Guangzhou Eighth People's Hospital, and informed consent was obtained from all patients enrolled.

\section{Definitions}

According to the Chinese diagnosis and treatment guideline for COVID-19 (trial version 7.0) [6], 288 patients were divided into non-severe group (258 cases), including light and general patients, and severe group (30 cases), including severe and critical patients. A case was defined as severe if it met any of the following: (1) shortness of breath, respiratory rate $\geq 30$ times / minute; (2) blood oxygen saturation $\leq 93 \%$ at rest; (3) oxygenation index $(\mathrm{PaO} 2 / \mathrm{FiO} 2) \leq 300 \mathrm{mmHg}$; (4) pulmonary infiltrates $>50 \%$ of the lung lesions within 24-48 hours; (5) respiratory failure, requiring mechanical ventilation; (6) shock; (7) combine with multiple organ dysfunction, needing ICU monitoring treatment. Acute Respiratory Distress Syndrome (ARDS) was defined according to WHO's guidance for COVID-19 [30]. Acute renal injury (ARI) was determined from serum creatinine [31]. Acute cardiac injury (ACI) was determined based on the serum concentration of troponin I (TNI) [11]. The reference ranges of all laboratory inspection indicators were measured in the laboratory of Guangzhou Eighth People's Hospital.

\section{Data collection}

This study reviewed the clinical electronic medical records, nursing records, laboratory tests and radiological findings of 288 adult patients with COVID19 , who were confirmed by nucleic acid testing. And we extracted epidemiology, demographics, clinical manifestations, laboratory data, chest radiography findings, treatment and outcome data for statistical analysis and research.

\section{Statistical analysis}

The purpose of this study was to analyze the risk factors of severe patients by comparing severe group and nonsevere group in terms of their clinical data. Therefore, no formal assumptions were used to facilitate the calculation of the sample size, and we included the largest number of patients who met the inclusion criteria.

We represented continuous variables as median and interquartile range (IQR), and categorical variables as 
frequency $(\mathrm{N})$ and percentage (\%). We assessed differences between severe group and non-severe group using two-sample $t$ test or the Mann-Whitney $\mathrm{U}$ test depending on parametric or nonparametric data for continuous variables, and $\chi 2$ test or Fisher's exact test for categorical variables. Univariate and multivariate logistic regression models were used to explore the risk factors for severe cases.

A P value of less than 0.05 was considered statistically significant. All data were statistically analyzed using SPSS software (version 25).

\section{AUTHOR CONTRIBUTIONS}

Dr. Feng He, Dr. Qingqing Luo and Dr. Ming Lei contributed equally, they all developed conceptualization and wrote the manuscript under the supervision of Dr. Na Yu, Dr. Liuping Yang and Dr. Jie Cao. Lixin Fan, Xinning Shao, Guanglie Huang, Jun Zeng, Ziwen Zhao, Shuguang Qin, Zhi Yang has participated substantially in the conceptualization and design of this work as well as the writing of the manuscript. All authors have reviewed the final version of the manuscript and have approved it for publication.

\section{ACKNOWLEDGMENTS}

We thank the patients, the nurses and physicians who provided care for the patients in Guangzhou Eighth People's Hospital, and the investigators in Guangzhou First People's Hospital.

\section{CONFLICTS OF INTEREST}

We declare no conflicts of interest.

\section{FUNDING}

This work was supported by grants from Natural Science Foundation of Guangdong Province (Grant no. 2017A030311035), and Fundamental Research Funds for the Central Universities (Grant no. y2eyD2191470).

\section{Editorial note}

${ }^{\&}$ This corresponding author has a verified history of publications using the personal email addresses for correspondence.

\section{REFERENCES}

1. Phelan AL, Katz R, Gostin LO. The novel coronavirus originating in Wuhan, China: challenges for global health governance. JAMA. 2020. [Epub ahead of print]. https://doi.org/10.1001/jama.2020.1097 PMID: $\underline{1999307}$

2. Zhu $\mathrm{N}$, Zhang $\mathrm{D}$, Wang $\mathrm{W}$, Li X, Yang $\mathrm{B}$, Song J, Zhao $\mathrm{X}$, Huang B, Shi W, Lu R, Niu P, Zhan F, Ma X, et al, and China Novel Coronavirus Investigating and Research Team. A novel coronavirus from patients with pneumonia in China, 2019. N Engl J Med. 2020; 382:727-33.

https://doi.org/10.1056/NEJMoa2001017 PMID: $\underline{31978945}$

3. Zhou F, Yu T, Du R, Fan G, Liu Y, Liu Z, Xiang J, Wang Y, Song B, Gu X, Guan L, Wei Y, Li H, et al. Clinical course and risk factors for mortality of adult inpatients with COVID-19 in Wuhan, China: a retrospective cohort study. Lancet. 2020; 395:1054-62. https://doi.org/10.1016/S0140-6736(20)30566-3 PMID: $\underline{32171076}$

4. Wu JT, Leung K, Leung GM. Nowcasting and forecasting the potential domestic and international spread of the 2019-nCoV outbreak originating in Wuhan, China: a modelling study. Lancet. 2020; 395:689-97. https://doi.org/10.1016/S0140-6736(20)30260-9 PMID:32014114

5. COVID-19 cases worldwide. June 3, 2020. https://www.worldometers.info/coronavirus/

6. National Health Commission of the People's Republic of China. Chinese diagnosis and treatment guideline for COVID-19 (trial version 7.0). March 4, 2020. http://www.nhc.gov.cn/xcs/zhengcwi/202003/46c929 4a7dfe4cef80dc7f5912eb1989.shtml

7. Wang $D$, Hu B, Hu C, Zhu F, Liu X, Zhang J, Wang B, Xiang $\mathrm{H}$, Cheng $Z$, Xiong $\mathrm{Y}$, Zhao $\mathrm{Y}$, Li Y, Wang $X$, Peng $Z$. Clinical characteristics of 138 hospitalized patients with 2019 novel coronavirus-infected pneumonia in Wuhan, China. JAMA. 2020; 323:1061-69.

https://doi.org/10.1001/iama.2020.1585 PMID: $\underline{2031570}$

8. Yang $X, Y u Y, X u J$, Shu $H, X i a J$, Liu $H$, Wu Y, Zhang L, Yu $Z$, Fang $M, Y u T$, Wang $Y$, Pan $S$, et al. Clinical course and outcomes of critically ill patients with SARS-CoV-2 pneumonia in Wuhan, China: a single-centered, retrospective, observational study. Lancet Respir Med. 2020; 8:475-81.

https://doi.org/10.1016/S2213-2600(20)30079-5 PMID:32105632

9. Chan JF, Yuan S, Kok KH, To KK, Chu H, Yang J, Xing F, Liu J, Yip CC, Poon RW, Tsoi HW, Lo SK, Chan KH, et al. A familial cluster of pneumonia associated with the 2019 novel coronavirus indicating person-to-person transmission: a study of a family cluster. Lancet. 2020; 395:514-23. 
https://doi.org/10.1016/S0140-6736(20)30154-9 PMID:31986261

10. Chen N, Zhou M, Dong X, Qu J, Gong F, Han Y, Qiu Y, Wang J, Liu Y, Wei Y, Xia J, Yu T, Zhang X, Zhang L. Epidemiological and clinical characteristics of 99 cases of 2019 novel coronavirus pneumonia in Wuhan, China: a descriptive study. Lancet. 2020; 395:507-13. https://doi.org/10.1016/S0140-6736(20)30211-7 PMID:32007143

11. Huang $C$, Wang $Y$, Li $X$, Ren L, Zhao J, Hu Y, Zhang L, Fan G, Xu J, Gu X, Cheng Z, Yu T, Xia J, et al. Clinical features of patients infected with 2019 novel coronavirus in Wuhan, China. Lancet. 2020; 395:497-506. https://doi.org/10.1016/S0140-6736(20)30183-5 PMID:31986264

12. Su YCF, Anderson $D E$, Young $B E$, Linster $M$, Zhu $F$, Jayakumar J, Zhuang Y, Kalimuddin S, Low JGH, Tan CW, Chia WN, Mak TM, Octavia S, et al. Discovery and Genomic Characterization of a 382-Nucleotide Deletion in ORF7b and ORF8 during the Early Evolution of SARSCoV-2. mBio. 2020; 11:e01610-20. https://doi.org/10.1128/mBio.01610-20 PMID:32694143

13. $X u X W, W u X X$, Jiang $X G, X u K J$, Ying $L, M a C L$, Li SB, Wang HY, Zhang S, Gao HN, Sheng JF, Cai HL, Qiu YQ, Li $\sqcup$. Clinical findings in a group of patients infected with the 2019 novel coronavirus (SARS-cov-2) outside of Wuhan, China: retrospective case series. BMJ. 2020; 368:m606. https://doi.org/10.1136/bmj.m606 PMID:32075786

14. Shen Z, Xiao Y, Kang L, Ma W, Shi L, Zhang L, Zhou Z, Yang J, Zhong J, Yang D, Guo L, Zhang G, Li H, et al. Genomic diversity of SARS-CoV-2 in coronavirus disease 2019 patients. Clin Infect Dis. 2020. [Epub ahead of print].

https://doi.org/10.1093/cid/ciaa203

PMID: $\underline{32129843}$

15. Hong KH, Choi JP, Hong SH, Lee J, Kwon JS, Kim SM, Park SY, Rhee JY, Kim BN, Choi HJ, Shin EC, Pai H, Park SH, Kim SH. Predictors of mortality in middle east respiratory syndrome (MERS). Thorax. 2018; 73:286-89.

https://doi.org/10.1136/thoraxjnl-2016-209313 PMID:28724637

16. Choi KW, Chau TN, Tsang O, Tso E, Chiu MC, Tong WL, Lee PO, Ng TK, Ng WF, Lee KC, Lam W, Yu WC, Lai JY, Lai ST, and Princess Margaret Hospital SARS Study Group. Outcomes and prognostic factors in 267 patients with severe acute respiratory syndrome in hong kong. Ann Intern Med. 2003; 139:715-23. https://doi.org/10.7326/0003-4819-139-9-200311040$\underline{00005}$ PMID: $\underline{14597455}$
17. Li P, Chen L, Liu Z, Pan J, Zhou D, Wang H, Gong H, Fu Z, Song $Q$, Min $Q$, Ruan $S, X u T$, Cheng $F$, Li X. Clinical features and short-term outcomes of elderly patients with COVID-19. Int J Infect Dis. 2020; 97:245-50.

https://doi.org/10.1016/j.ijid.2020.05.107 PMID: $\underline{2492533}$

18. Zhao $M$, Wang $M$, Zhang J, Gu J, Zhang $P, X u Y, Y e J$, Wang Z, Ye D, Pan W, Shen B, He H, Liu M, et al. Comparison of clinical characteristics and outcomes of patients with coronavirus disease 2019 at different ages. Aging (Albany NY). 2020; 12:10070-86.

https://doi.org/10.18632/aging.103298 PMID: 32499448

19. Shahid Z, Kalayanamitra R, McClafferty B, Kepko D, Ramgobin D, Patel R, Aggarwal CS, Vunnam R, Sahu N, Bhatt D, Jones K, Golamari R, Jain R. COVID-19 and older adults: what we know. J Am Geriatr Soc. 2020; 68:926-29. https://doi.org/10.1111/igs.16472 PMID:32255507

20. Zhang $\mathrm{H}$, Kang Z, Gong H, Xu D, Wang J, Li Z, Cui X, Xiao J, Meng T, Zhou W, Liu J, Xu H. The digestive system is a potential route of 2019-nCov infection: a bioinformatics analysis based on single-cell transcriptomes. bioRxiv. https://doi.org/10.1101/2020.01.30.927806

21. Wong SH, Lui RN, Sung JJ. Covid-19 and the digestive system. J Gastroenterol Hepatol. 2020; 35:744-48. https://doi.org/10.1111/igh.15047 PMID:32215956

22. Shang J, Ye G, Shi K, Wan Y, Luo C, Aihara H, Geng Q, Auerbach A, Li F. Structural basis of receptor recognition by SARS-CoV-2. Nature. 2020; 581:221-24. https://doi.org/10.1038/s41586-020-2179-y PMID: $\underline{32225175}$

23. Zou X, Chen K, Zou J, Han P, Hao J, Han Z. Single-cell RNA-seq data analysis on the receptor ACE2 expression reveals the potential risk of different human organs vulnerable to 2019-nCoV infection. Front Med. 2020; 14:185-92. https://doi.org/10.1007/s11684-020-0754-0 PMID: 32170560

24. Oudit GY, Kassiri Z, Jiang C, Liu PP, Poutanen SM, Penninger JM, Butany J. SARS-coronavirus modulation of myocardial ACE2 expression and inflammation in patients with SARS. Eur J Clin Invest. 2009; 39:618-25. https://doi.org/10.1111/i.1365-2362.2009.02153.x PMID: 19453650

25. Li JW, Han TW, Woodward M, Anderson CS, Zhou H, Chen YD, Neal B. The impact of 2019 novel coronavirus on heart injury: a systematic review and meta-analysis. Prog Cardiovasc Dis. 2020; S0033-0620:30080-83. https://doi.org/10.1016/i.pcad.2020.04.008 PMID:32305557 
26. McRae MP, Simmons GW, Christodoulides NJ, Lu Z, Kang SK, Fenyo D, Alcorn T, Dapkins IP, Sharif I, Vurmaz D, Modak SS, Srinivasan K, Warhadpande S, et al. Clinical decision support tool and rapid point-of-care platform for determining disease severity in patients with COVID-19. Lab Chip. 2020; 20:2075-85. https://doi.org/10.1039/d0lc00373e PMID:32490853

27. Jung HY, Lim JH, Kang SH, Kim SG, Lee YH, Lee J, Chang $\mathrm{HH}$, Kim SW, Choi JY, Cho JH, Kim CD, Kim YL, Park SH. Outcomes of COVID-19 among patients on in-center hemodialysis: an experience from the epicenter in South Korea. J Clin Med. 2020; 9:E1688.

https://doi.org/10.3390/jcm9061688

PMID:32498262

28. Zhang F, Liang Y. Potential risk of the kidney vulnerable to novel coronavirus 2019 infection. Am J Physiol Renal Physiol. 2020; 318:F1136-37.

https://doi.org/10.1152/ajprenal.00085.2020

PMID:32223555

29. Guan WJ, Ni ZY, Hu Y, Liang WH, Ou CQ, He JX, Liu L, Shan H, Lei CL, Hui DSC, Du B, Li LJ, Zeng G. China Medical Treatment Expert Group for Covid-19. Clinical
Characteristics of Coronavirus Disease 2019 in China. N Engl J Med. 2020; 382:1708-1720.

https://doi.org/10.1056/NEJMoa2002032

PMID:32109013

30. WHO Clinical management of severe acute respiratory infection when novel coronavirus (nCoV) infection is suspected. Jan 11, 2020. https://www.who.int/ publications-detail/clinical-management-of-severeacute-respiratory-infection-when-novel-coronavirus(ncov)-infection-is-suspected

31. Khwaja A. KDIGO clinical practice guidelines for acute kidney injury. Nephron Clin Pract. 2012; 120:c179-84. https://doi.org/10.1159/000339789 PMID:22890468 